\title{
Treatment of juvenile angiofibromas: 18-year experience of a single tertiary centre in Spain*
}

\author{
Fernando López, Vanessa Suárez, María Costales, Carlos Suárez, \\ José L. Llorente
}

Department of Otorhinolaryngology, Instituto Universitario de Oncología del Principado de Asturias,

Hospital Universitario Central de Asturias, Oviedo, Asturias, Spain
Rhinology 50: 95-103, 2012

DOI: $10.4193 /$ Rhino.11.129

*Received for publication:

June 19, 2011

accepted: November 4, 201

\section{Summary}

Background: The management of juvenile angiofibroma (JA) has changed during the last decades but it still continues to be a challenge. The objective of this study was to review the used treatment and our outcomes.

Methods: From 1992 to 2010, 48 cases of JA were treated at our department. Charts were reviewed for standard demographic, tumour size and location, vascular supply and results of embolization, surgical approach, operative results, adjuvant therapies, recurrence and postoperative follow-up.

Results: Most tumours were Andrews-Fisch stages III and IV and surgery was used as the main treatment in all cases. We used an open surgical approach in 37 (77\%) patients and 11 (23\%) were treated endoscopically. The most common open approach used in this series was the subtemporal-preauricular approach. Until 1995, all tumours were operated on by a conventional open approach. Afterwards, early-stage tumours were operated on through an endoscopic approach. Ten patients were treated through surgery followed by radiosurgery. Two (4\%) patients had recurrent disease.

Conclusions: These tumours should be treated at centres with expertise in skull base surgery to achieve complete surgical resections with low morbidity. Radiosurgery after surgery seems to be a valuable option in the long-term control of some extended JAs.

Key words: juvenile angiofibroma, treatment, radiosurgery, surgery, outcomes, and recurrence

\section{Introduction}

Juvenile angiofibromas (JAs) are uncommon and highly vascular tumours that are observed mostly in adolescent male patients and account for less than $0.5 \%$ of all head and neck tumours. Recent analysis of JAs show tumour's genetic abnormalities to be involved in their origin and provide evidence for a role of androgens in JAs biology ${ }^{(1)}$. JAs originate from the superior posterior lip of the sphenopalatine foramen and despite its benign histopathologic nature, these tumours have tendency for orbit and intracranial extension and skull base erosion. Intracranial extension was reported in 10 to $36 \%$ of all cases and it is almost always extradural.
JAs have been managed according to various protocols, including surgery and radiotherapy. Complete surgical extirpation remains the mainstay of treatment. The rate of radical cure of early stage JAs is approximately $100 \%$ using non-invasive approaches. The endoscopic surgery due to its minimal invasiveness, low morbidity rate, and low rates of recurrence, has caused a paradigm shift in the surgical treatment of the JAs. Endoscopic resection has allowed the radical removal of selected JAs. However, in up to $40 \%$ of cases, the tumour is diagnosed in advanced-stage. In these extended JAs (Andrews-Fisch III and IV) management is still a surgical challenge and, despite of the development of preoperative tumour embolization and that endoscopic resection of large or extended tumour is feasible in 
expert hands, open approaches play an important role. These procedures provide the ability to resect large tumours but the potential mortality and morbidity associate with these procedures is relatively high and may still leave residual disease.

This article presents a retrospective, descriptive study of patients with a diagnosis of JA treated at our tertiary care academic teaching hospital during the past 18 years. We present our results in the surgical treatment of these lesions considering tumour stage and paying attention to surgical strategy, results and recurrences. We emphasize the introduction of endoscopic techniques in the routine approach of JAs and adjuvant treatment with radiosurgery.

\section{Patients and methods}

Study design

The surgical medical charts of the Otorhinolaryngology Department of our hospital, from 1992 to May 2010, were reviewed to collect data regarding the clinical data of patients diagnosed with JAs. The methodology was a retrospective, non-randomized chart review. We identified 48 patients diagnosed and treated of JA. All patients gave their signed informed consent, and the study had received prior approval from the ethics committee of our institution.

\section{Data collection}

Data collection was based on a review of the patients medical histories, recorded data on age, tumour size, laterality, location, staging, presenting symptoms, vascular supply, results of embolization, surgical procedures, blood loss, intraoperative and postoperative complications, perioperative morbidity, recurrence, and status at last follow-up visit. Extent of the tumour was determined by evaluation of patient's paranasal computed tomography (CT) and/or and magnetic resonance imaging (MRI). CT scan showed erosion of the skull base and MRI scans were useful to demonstrate the intracranial and intraorbital extension. Skull base involvement was defined as radiographic evidence of bone erosion of the skull base excluding the pterygoid plates, anterior wall of sphenoid sinus, orbital walls, and maxillary sinus walls. Tumours were classified according to their location and growth pattern using the Andrews-Fisch system (Table 1).

\section{Patient follow-up}

The follow-up consisted of periodic visits to our clinic. During these visits, the patients were asked about any suspicious symptoms and were examined with a nasal endoscope. A CT and/or MRI were performed annually in all patients for the first 5 years of follow-up. After that, radiographic studies were only performed if the patient developed new symptoms. Local control was defined as absence of any radiographic or endoscopic abnormalities. In addition, patients with residual static or resolving abnormalities over repeated imaging were considered to be locally controlled. Duration of local control was calculated from date of surgery. The average follow-up period was 8 years (range 3 to 18 years).

\section{Results}

The study group consisted of 47 men and 1 woman, with an average age at diagnosis of 16 years (range 11 to 18 years). Diagnosis was confirmed with postoperative histopathologic examination in all patients. The woman patient was confirmed as female by gynaecological examination. She showed a normal female karyotype $(46, X X)$, which was subsequently confirmed by her motherhood; pathological findings revised were consistent with angiofibroma. Fourteen patients had previous incomplete resections performed elsewhere, before being referred to our department.

The tumours were located on the right side in 29 patients (60\%) and on the left side in 19 patients (40\%). The mean duration from the onset of symptoms until first surgery was 14 months (1 to 84 months). The most common presenting symptoms were nasal airway obstruction in $36 / 48$ patients $(75 \%)$ and epistaxis in $33 / 48$ (68\%). One patient with stage IVa JA was presented with severe left visual acuity deterioration due to optic nerve compression. Different symptoms are illustrated in Table 2.

Angiography was performed in 44 (92\%) patients. Four (8\%) patients did not undergo angiography due to technical reasons. The vessels feeding the tumours were identified as the external carotid system (external carotid artery, ascending pharyngeal artery or middle meningeal artery) in 35 patients (80\%), as the external and internal carotid systems in 8 patients (18\%) and as the internal carotid system only in 1 patient (2\%). Six of $44(14 \%)$ patients had tumours with bilateral vascular supply. Selective embolization 24 - 48 hours before surgery was performed in 37 cases (77\%) with Polyvinyl Alcohol (PVA) particles. In cases with significant vascular supply originated from the internal carotid system, embolization could not be performed.

All JAs were located in the nasal cavity and nasopharyngeal area. Most tumours (90\%) showed extension to the pterygopalatine fossa. Twenty-two cases (46\%) extended intracranial, 8 tumours (17\%) extended intradurally and in one case the cavernous sinus was infiltrated. Most patients (73\%) had advanced JAs (stages III and IV). The tumour stage stratified by surgical approach is shown in Table 3.

All patients were treated with surgery. The surgical approach used in each case was typically chosen depending on the stage and extension of the tumour and personal experience of the surgeon. Until 1995, all tumours were operated on by the conventional open approach. Afterwards, early tumour stages (I and II) were operated on through an endoscopic approach. We used an 
Table 1. Andrew's (modified Fisch) classification.

\begin{tabular}{|ll}
\multicolumn{1}{c|}{ Stage } & \multicolumn{1}{c}{ Description } \\
\hline Stage I & $\begin{array}{l}\text { Limited to the nasopharynx and nasal cavity, bone } \\
\text { destruction negligible, or limited to the sphen- } \\
\text { opalatine foramen }\end{array}$ \\
Stage II & $\begin{array}{l}\text { Invades the pterygopalatine fossa or the maxillary, } \\
\text { ethmoid or sphenoid sinus with bone destruction }\end{array}$ \\
Stage IIIa & $\begin{array}{l}\text { Invades the infratemporal fossa or the orbital } \\
\text { region without intracranial involvement }\end{array}$ \\
Stage IIIb & $\begin{array}{l}\text { Invades the infratemporal fossa or orbit with } \\
\text { intracranial extradural involvement }\end{array}$ \\
Stage IVa & $\begin{array}{l}\text { Intracranial intradural tumour without infiltration } \\
\text { of the cavernous sinus, pituitary fossa, or optic } \\
\text { chiasm }\end{array}$ \\
\hline Stage IVb & $\begin{array}{l}\text { Intracranial intradural tumour with infiltration } \\
\text { of the cavernous sinus, pituitary fossa, or optic } \\
\text { chiasm }\end{array}$ \\
\hline
\end{tabular}

open surgical approach in 37 (77\%) patients and 11 (23\%) cases were treated endoscopically. The most commonly used open approach was the subtemporal-preauricular infratemporal approach, which was performed in 14 (29\%) cases. Anterior facial translocation was performed in $13(27 \%)$ cases (7 patients were approached through midfacial degloving techniques, 2 patients through lateral rhinotomy incisions and 4 patients through midfacial degloving and hemicoronal-preauricular incision). Conventional lateral facial translocation was performed in $5(10 \%)$ cases, transmaxillary approach (lateral rhinotomy approach) in 2 (4\%) cases and subcraneal approach in $3(6 \%)$ cases.

In 9 cases (3 subtemporal-preauricular infratemporal approach, 3 lateral facial translocation and 3 anterior facial translocation with hemicoronal preauricular incision) surgical reconstruction was carried out using a temporalis muscle flap. The aesthetic deformity produced in the temporal region by the rotation of the muscle was corrected using the anterior rotation of the posterior half of the muscle.

The operative results for all patients are shown in Table 4. There was no surgery-related mortality. Preoperative symptoms improved significantly in all patients except the decrease in visual acuity observed in one patient classified as IVa. Intraoperative blood loss varied from 150 to $8000 \mathrm{~mL}$ (mean, $2217 \mathrm{~mL}$ ). Intraoperative blood transfusion was required in 38 (79\%) patients (only 3 patients had been operated on by endoscopic approach). A significant reduction in blood loss and transfusion was seen moving from open surgery to endoscopic surgery. Patients with
Table 2. Presenting symptoms in 48 patients with juvenile angiofibroma.

\begin{tabular}{|lc|}
\hline \multicolumn{1}{c}{ Symptom } & No. of patients (\%) \\
\hline Nasal airway obstruction & $36(75)$ \\
\hline Epistaxis & $33(68)$ \\
\hline Nasal discharge & $9(19)$ \\
\hline Hyposmia/anosmia & $6(13)$ \\
\hline Hearing loss & $5(10)$ \\
\hline Epiphora & $3(6)$ \\
\hline Eye proptosis & $1(2)$ \\
Facial swelling & $5(10)$ \\
\hline Otitis media with effusion & $5(10)$ \\
\hline Rhinolalia & $2(4)$ \\
\hline Diplopia & $1(2)$ \\
\hline Headache & $4(8)$ \\
Amaurosis & $1(2)$ \\
\hline Snoring & $1(2)$ \\
\hline
\end{tabular}

Patients usually presented with more than one symptom.

advanced-stage tumours (III and IV) had higher intraoperative bleeding compared with those with early-stage tumours (I and II) $(p<0.001)$. The large volume losses were more likely in the setting of open approaches (mean, $2833 \mathrm{~mL}$ ) and relatively uncommon with strictly endoscopic resections (mean, $895 \mathrm{~mL})(p<0.001)$.

There were no complications in patients undergoing endoscopy. Dehiscence and infection of the surgical wound were seen in one patient who underwent a lateral facial translocation (stage IIlb) and in one patient who underwent a subtemporalinfratemporal preauricular approach (stage IIla). Two cases of temporary abducens nerve palsy that recovered spontaneously within 6 months were encountered in stage Illb tumours. Finally, severe trimus (patient underwent subtemporal-infratemporal preauricular approach) and lacrimal duct dysfunction (2 patients underwent lateral facial translocation and other lateral rhinotomy) were observed in 3 patients.

The hospital stay was a mean of 8 days in those who had an endoscopic resection and 15 days in those who had an open resection $(p<0.001)$.

Total removal of the tumour was always the initial goal of surgery. A macroscopic complete tumour resection was achieved in 38 (79\%) patients, which was confirmed during postoperative and follow-up imaging. In 10 (21\%) cases (all stage IV), immediate postoperative MRI studies revealed residual tumour near the cavernous sinus. Because the high likelihood of producing significant morbidity and due to JAs are benign lesions, tumour 
Table 3. Andrews-Fisch stage tumour by surgical approach.

\begin{tabular}{|c|c|c|c|c|c|c|c|}
\hline \multirow[b]{2}{*}{ Stage } & \multicolumn{7}{|c|}{ Surgical approach } \\
\hline & AFT & SP & LFT & TM & SC & $\begin{array}{c}\text { Endoscopic ap- } \\
\text { proach }\end{array}$ & All \\
\hline 1 & 0 & 0 & 0 & 0 & 0 & 1 & $1(2 \%)$ \\
\hline II & 1 & 0 & 1 & 0 & 0 & 10 & $12(25 \%)$ \\
\hline Illa & 6 & 5 & 1 & 1 & 0 & 0 & $13(27 \%)$ \\
\hline IIIb & 4 & 7 & 2 & 1 & 0 & 0 & $14(29 \%)$ \\
\hline IVa & 2 & 1 & 1 & 0 & 3 & 0 & $7(15 \%)$ \\
\hline $\mathrm{IVb}$ & 0 & 1 & 0 & 0 & 0 & 0 & $1(2 \%)$ \\
\hline
\end{tabular}

$\mathrm{AFT}=$ anterior facial translocation; $\mathrm{SP}=$ subtemporal-preauricular infratemporal approach; LFT = lateral facial translocation; $\mathrm{TM}=$ transmaxillary approach; SC = subcranial approach

fragments located near the orbital apex, into the cavernous sinus or attached to the internal carotid artery were not removed avoiding the mobilization of critical neurovascular structures. All these patients had been operated on via the open approach. The mean residual volume was $3.7 \mathrm{~cm}^{3}$ (range, $2.9-5 \mathrm{~cm}^{3}$ ). Between 3 and 7 months after surgery, patients with residual tumour were treated with radiosurgery. Four patients underwent GammaKnife ${ }^{\oplus}$ surgery and 6 patients fractionated stereotactic radiotherapy. Patients tolerated treatment without acute complications. However, 2 patients developed cataracts 2 years after treatment. Regular follow-up MRI study was done annually and revealed no evidence of disease in 1 patient, marked decrement of the size of tumour in 2 patients and no change in 7 patients, after a 3-year minimum follow-up (Table 5).

JA recurrence has to be considered, when clinical and radiological tumour suspicious correlates with clinical symptoms and growth during follow-up. In our series, 2 (4\%) patients (stage IIIb) had recurrent disease following the surgery. These lesions were discovered at 16 and 28 months, respectively. Both patients had been previously operated on via the open approach. In one case, the recurrence was located in the infratemporal fossa and was operated on via the subtemporal-infratemporal preauricular approach. In the other case, the recurrence affected the nasopharynx and the contralateral pterygoid plate base and it was removed via the endoscopic approach. These patients are currently free of disease and asymptomatic.

\section{Discussion}

$J A$ is an infrequent diagnosis whose management is usually performed at centres with expertise in skull base surgery to achieve best results. For this reason, most series in the literature are relatively small (median 30 , mean 33 , range $12-95)^{(2)}$.
$\mathrm{JA}$ is a benign tumour that occurs in adolescent males almost exclusively. In our study, except in one case, all the patients were men with a mean age at diagnosis of 16 years, which is similar from reports of other authors ${ }^{(3)}$. Although isolated cases in females have been well described, due to its exceptional occurrence, we recommend a comprehensive review of the pathologic study and conducting genetic testing.

The most common clinical presentation includes nasal obstruction and recurrent epistaxis ${ }^{(4)}$. In our study, more than half of the patients had some of these symptoms at the time of diagnosis. As the tumour progresses and affects the orbit, the skull base or causes expansion of the sinonasal cavities, physical examination may reveal facial deformity, proptosis, palate expansion and visual or neurologic impairment. Currently, due to improved imaging techniques, patients present with earlier stages and compressive and neurological symptoms are less frequent. Our patients were evaluated with a median interval after symptom onset of 14 months. Sometimes their delay in presentation can be attributed to a tendency to associate the indolent symptoms of JA with more common disease entities such as rhinitis, sinusitis and antrochoanal polyps.

Table 4. Operative results: All juvenile nasopharyngeal angiofibroma patients.

\begin{tabular}{lcc} 
& $\begin{array}{c}\text { Open } \\
\text { approach }\end{array}$ & $\begin{array}{c}\text { Endoscopic } \\
\text { approach }\end{array}$ \\
\hline Mean blood loss (mL) & 2833 & 895 \\
Transfusion (No. patients) & 35 & 3 \\
$\begin{array}{l}\text { Residual tumour after } \\
\text { surgery (No. patients) }\end{array}$ & 10 & 0 \\
Recurrence & 2 & 0
\end{tabular}


Table 5. Juvenile nasopharyngeal angiofibroma. Radiosurgery treatment. Outcomes.

\begin{tabular}{|c|c|c|c|c|c|c|}
\hline Cases & $\begin{array}{c}\text { Location of residual } \\
\text { tumour }\end{array}$ & Size $\left(\mathrm{cm}^{3}\right)$ & Radiosurgery & $\begin{array}{l}\text { Months between sur- } \\
\text { gery and radiotherapy }\end{array}$ & Follow-up & Current status \\
\hline Case 1 & CS (bilateral) & 5 & $\begin{array}{l}\text { FSRT (50 Gy; } \\
2 \text { Gy/fraction) }\end{array}$ & 3 & 180 & NED \\
\hline Case 2 & CS & 3.1 & $\begin{array}{l}\text { FSRT (35 Gy; } \\
2 \text { Gy/fraction) }\end{array}$ & 2 & 156 & NC \\
\hline Case 3 & CS & 1.8 & $\begin{array}{c}\text { GKRS (14 Gy; } \\
50 \% \text { isodose line) }\end{array}$ & 3 & 144 & DS \\
\hline Case 4 & CS & 3 & $\begin{array}{c}\text { GKRS (20 Gy; } \\
45 \% \text { isodose line) }\end{array}$ & 4 & 120 & NC \\
\hline Case 5 & CS & 4.5 & $\begin{array}{c}\text { GKRS (20 Gy; } \\
55 \% \text { isodose line) }\end{array}$ & 4 & 85 & NC \\
\hline Case 6 & CS (bilateral) & 5.1 & $\begin{array}{c}\text { FSRT (38 Gy; } \\
1.8 \mathrm{~Gy} / \text { fraction) }\end{array}$ & 4 & 67 & NC \\
\hline Case 7 & CS & 4.2 & $\begin{array}{l}\text { FSRT (50 Gy; } \\
2 \text { Gy/fraction) }\end{array}$ & 7 & 48 & NC \\
\hline Case 8 & CS & 4.1 & $\begin{array}{l}\text { FSRT (50 Gy; } \\
2 \text { Gy/fraction) }\end{array}$ & 4 & 46 & DS \\
\hline Case 9 & CS & 3.2 & $\begin{array}{l}\text { FSRT (50 Gy; } \\
2 \text { Gy/fraction) }\end{array}$ & 3 & 47 & NC \\
\hline Case 10 & CS & 2.9 & $\begin{array}{c}\text { GKRS ( } 26 \mathrm{~Gy} \\
55 \% \text { isodose line) }\end{array}$ & 2 & 40 & NC \\
\hline
\end{tabular}

$\mathrm{CS}=$ cavernous sinus region; FSRT = fractionated stereotactic radiotherapy/radiosurgery; GKRS = GammaKnife ${ }^{\oplus}$ radiosurgery; NED = no evidence of disease; $\mathrm{DS}=$ decrement of size; $\mathrm{NC}=$ no change

Different staging systems have been proposed based on tumour extension ${ }^{(5)}$. We think that all systems have their pros and cons but any proposed system should be closely correlated with clinical and radiological findings. UPMC staging system ${ }^{(6)}$ incorporates important prognostic information for the surgeon such as the residual vascularity of the tumour following embolization and the route of skull base extension. This staging system provides a good prediction of morbidity (blood loss and need for multiple operations) and tumour recurrence. In our study, $73 \%$ of patients had an advanced stage and $46 \%$ had intracranial extension, slightly higher than those observed by other authors. This would be partially justified because our hospital is a tertiary referral centre for skull base pathology, and $48 \%$ of cases were referred from other centres and these usually are more advanced JAs.

The diagnosis of JA is based on a precise clinical history, examination of the patient, and imaging (CT and MRI). Preoperative tissue biopsies should be avoided due to the highly vascular nature of the tumour. Using CT, MRI imaging and angiography or angio-MRI, we can delineate the exact location of the tumour, determine the relationship of the tumour to important vascular and neurologic structures and define accurately the bloody supply to select the least traumatic approach with secure haemostatic control. CT delineates the depth of invasion into the cancellous bone of the sphenoid sinus, a main predictor of recurrence. MRI is especially critical in assessment of intracranial tumour extension and in the follow-up after treatment to detect recurrent and residual disease ${ }^{(7)}$.

Bilateral carotid angiography is required before surgery to outline the vascular supply to the tumour and perform, if it is possible, the embolization of the feeding vessels. Although most authors state that it is effective, a few others cast doubts about its validity and they propose that preoperative embolization distort tumour boundaries, leading to incomplete resection ${ }^{(8,9)}$. We recommend performing preoperative tumour embolization of the external carotid artery. The occlusion of the tumour feeder's reduces the intraoperative bleeding, a major source of morbidity, and it can lead to a tumour shrinkage, allowing an easier tumour removal and a better visualization of the surgical field during surgery (especially in endoscopic approaches) ${ }^{(10)}$.This increases the chances of complete surgical resection, which is key to preventing recurrences of disease as is reported by McCombe et al., ${ }^{(11)}$. The vascular supply comes mainly from the external carotid artery branches such as the internal maxillary artery, the ascending pharyngeal artery and the vidian artery, as seen in more than $80 \%$ of patients in our series. Sometimes advanced stage tumours are fed through branches of the internal carotid artery such as the ophthalmic artery, which should not be embolized because the high risk of complication. Furthermore, 


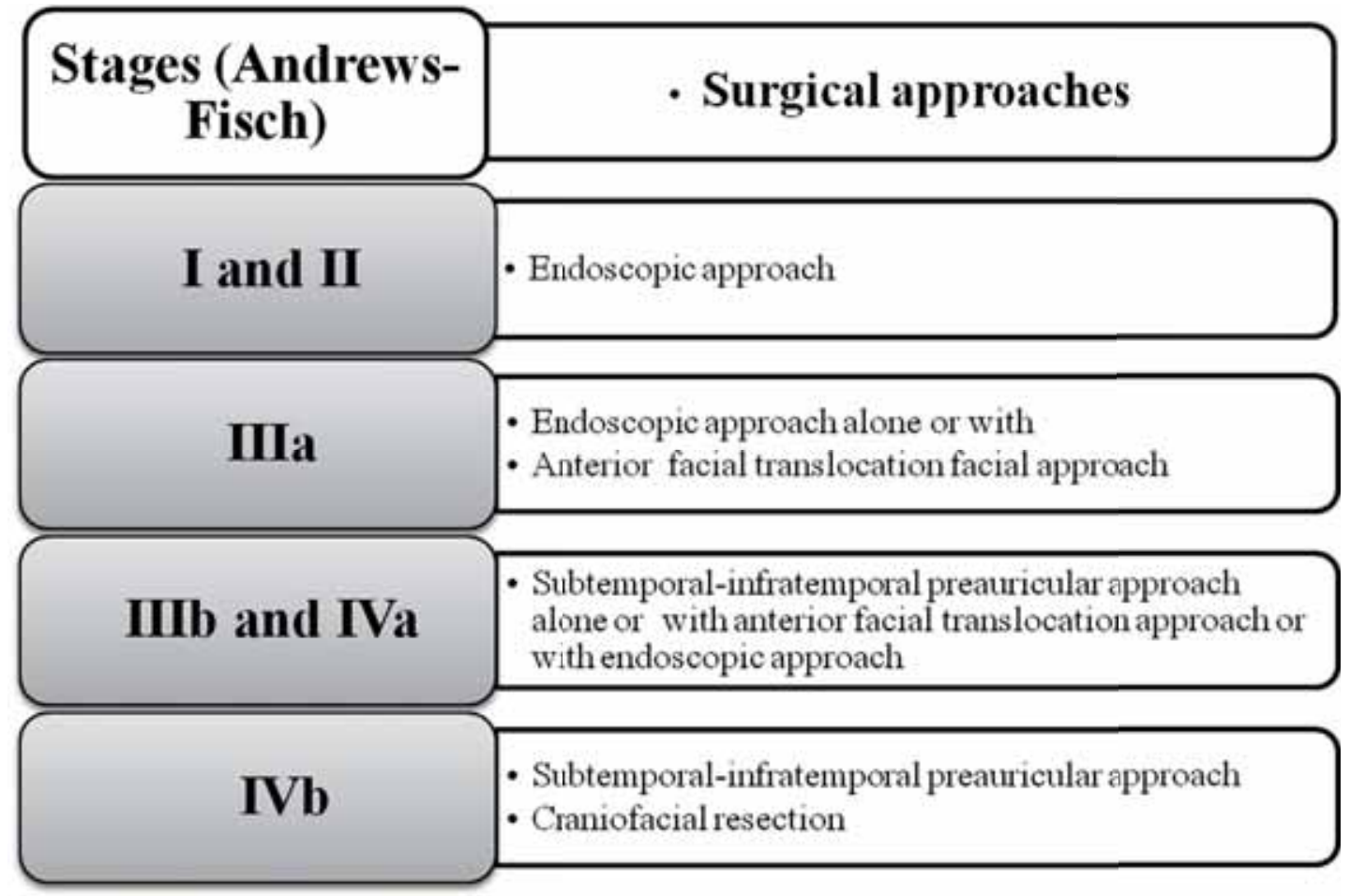

Figure 1. General scheme of treatment of JAs in our Department, according Andrews-Fisch stage.

when the cavernous sinus is involved, we can observe'neovascularization' from the internal carotid artery. Despite the risk of complications is higher, these 'new vessels' could be embolized. In our study, the internal carotid artery was involved in $20 \%$ of cases. Sometimes JA is supplied by both the left and the right external carotid arteries (18\% in our series). In these cases, bilateral internal maxillary artery embolization should be performed to prevent backflow by one of them and the formation of an alternative vascular supply, which favours haemorrhage. It is important to note that the blood supply to the temporalis muscle, which may be used for reconstruction, could be compromised by embolization. Embolization may carry a small additional risk (12) but we believe that it enhances the overall surgical safety. In this study, $77 \%$ of patients were embolized and there were no notable complications. If the preoperative tumour embolization cannot be performed, the reduction of intraoperative bleeding is achieved after control and ligation of feeding vessels (mainly internal maxillary artery) before tumour dissection begins.

Hypotensive general anaesthesia and the use of radiofrequency coblation help to decrease bleeding.

Most authors agree that surgery is the primary and exclusive treatment of uncomplicated primary and recurrent $\mathrm{JA}^{\left({ }^{(13)} \text {. The }\right.}$ extent of initial surgery is crucial to prevent recurrence. The choice of surgical approach is based on tumour size, extent and location. The surgeon's skills and experience are crucial for choosing the optimal approach because each one has advantages and limitations. Accordingly, more-experienced surgeons can perform more limited approaches to remove large tumours. However, the approach should allow wide enough exposure of the tumour for achieving complete resection and manage the eventual complications. Figure 1 shows the general surgical strategy used in our centre according to the stage.

Traditionally, JAs have been approached through open surgical approaches that include transoral, transfacial, lateral and craniofacial approaches ${ }^{(4,14)}$. Open approaches to JAs continue to be favoured and there is no doubt that for stages IIIlb and IV they remain indicated ${ }^{(14)}$. These procedures require facial incisions that carry the potential for anaesthetic facial scarring. In our department, the traditional transfacial approaches were performed in the older cases and since 1998 are no longer performed. We advocate the anterior facial translocation by means of a midfacial degloving and preauricular subtemporalinfratemporal approach for treatment of most of large JAs and, in our series, $56 \%$ of patients were operated on by these approaches. Both approaches have enabled us to avoid the sequelae associated with facial incisions typical of the conventional approaches. Facial translocation is the method of choice in large volume tumours. Its modular design allows great versatility, accommodating osteotomies at the location of the tumour, which facilitates good exposure of both the nasosinusal area as well as of the infratemporal fossa, while making the approach to the tumour possible from different perspectives (lateral and anterior-posterior) ${ }^{(15)}$. A disadvantage of this approach is relatively poor access to the skull base behind of the tumour 
and, although most intracranial lesions can be safely removed because the tumour grows with a pushing border that can be dissected from the dura relatively easily, the complete extirpation of these tumours may be difficult if bleeding is severe. In these cases an ipsilateral hemicoronal incision is needed to access securely the skull base. The preauricular subtemporalinfratemporal is used especially when the tumour has a limited anterior extension that nevertheless affects the infratemporal fossa towards the floor of the media fossa or the parasellar region ${ }^{(16)}$. This approach also permits bilateral access to the nasopharynx and permits ligation of the internal maxillary artery early in the dissection thus limiting intraoperative blood loss. We use this approach if JA is not too voluminous and affects the nasopharynx, the infratemporal fossa, and the floor of the media fossa or the parasellar region with little anterior progression. With bigger tumours, or when the tumour widely extends to the infratemporal fossa or the paranasal sinuses, we resort to a facial translocation approach by degloving. Sometimes preauricular approaches can be combined with an endoscopic approach to remove the tumour after detaching it at the top (17). Finally, we think that a craniofacial-subcranial approach is appropriate for some JAs that extend intracranially and affect the floor of anterior fossa, the sellar region or optic chiasm ${ }^{(18)}$. A review of these open procedures reported no evidence of arrest in facial and cranial growth in young patients ${ }^{(19)}$. Our study also confirms these findings.

Since the first reports about minimally invasive endoscopic resections were published, the endoscopic approach has become the approach of choice for select JAs ${ }^{(6,20-23)}$. The main advantage of the endoscopic approach relates to its low morbidity, shorter duration of hospitalization, and improved or equivalent recurrence rates, as seen in our series. Initially, the endoscopic approach was used for the management of small-sized tumours (stages I and II) but, currently, the increase endoscopic surgical skills has allowed to extend the indications of endoscopic approaches (stages IIla). Proper patient selection is essential for successful endoscopic management of JA. According to some authors, the lateral extension of tumour into the infratemporal fossa could be a limitation of this technique ${ }^{(14)}$. However, in our opinion, given the absence of adhesions of the tumour to surrounding tissues, it can pull the tumour into the nasal cavity relatively easily and safely. Since 1995, 11 of the 48 patients (23\%) underwent an endoscopic approach. All patients were in stage I or II and complete resection was possible in all patients without any complications. These results confirm those of other authors $(17,20,21,24)$.

Hormonal therapy ${ }^{(24)}$ and chemotherapy ${ }^{(25)}$ have been used but nearly abandoned because of ineffectiveness and significant side effects. Mishra et al., stated that spontaneous regression is only valuable for residual tumours ${ }^{(27)}$. We observed regression in one case after surgery and adjuvant radiosurgery. The use of radiotherapy as single treatment modality seems inappropriate because it is a benign tumour ${ }^{(26)}$. However, radiotherapy is advocated for patients who present residual or recurrent disease in critical anatomic areas or when the tumour involves vital structures with high surgical morbidity ${ }^{(28,29)}$. In our study 10 patients (21\%) were treated for advanced JA through surgery followed radiosurgery and, after a median follow-up of 8 years, control of disease was probed. All these patients had intracranial intradural invasion, so the rate of residual disease is slightly higher to that reported in other studies in advanced angiofibromas ${ }^{(30,31)}$. We believe that an appropiate strategy in the management of advanced JA is the surgical resection to shrink the tumour to a size suitable for treatment with radiosurgery in locations such as the internal carotid artery, cavernous sinus, optic nerve or middle fossa. The use of radiotherapy is still a matter of debate if radiation can be considered a safe therapeutic option in young patients who have a likelihood to develop growth impairment, acute or chronic central nervous system syndrome and sarcomatoid degeneration of residual JAs. In our department, we agree with Lee et al., who conclude that the risk of complications from radiation is less than the morbidity and possible mortality associated with surgical resection ${ }^{(29)}$. Önerci et al., suggest that a 'wait and see' approach may be preferable to craniofacial resection if there is residual intracranial tumour after surgery ${ }^{(32)}$. We agree that in case of patients with residual intracranial disease a second surgery is considered as the best option if the tumour can be reasonably extirpated. However, they should not be routinely operated because complete removal of the tumour from the cavernous sinus would cause a significant morbidity that hardly justified during excision of a benign tumour. Because JA are tumours arising in the young population, tumour remnants might grow during the patient's life time and cause uncontrollable haemorrhage and neurological deficits. Therefore we believe that residual tumour should be treated and radiosurgical procedure is a good option with acceptable morbidity. However, the roles of the 'wait and see' strategy and radiosurgery will require further prospective studies.

Complete removal of $\mathrm{JA}$ is essential to prevent recurrence and most, if not all, recurrences could be reflecting incomplete resection of the tumour. We agree with Howard et al., who emphasize the importance of residual disease in the basisphenoid and the pterygoid canal as an indicator of recurrence ${ }^{(33)}$. Lloyd et al., found that in $60 \% \mathrm{JAs}$ expanded posteriorly along the pterygoid canal with invasion of the cancellous bone of the sphenoid and they observed that $93 \%$ of recurrences occurred in patients with invasion of sphenoid diploe ${ }^{(7)}$. Meticulous subperiostial dissection and drilling of these areas is necessary to ensure complete removal and to avoid recurrences. Recurrence rates of 
on average $32 \%$ have been described ${ }^{(2)}$. Endoscopic recurrence rates have generally been lower but this may be attributable to the bias that early-stage tumours are more likely to be treated endoscopically but, in general endoscopic resection has equivalent recurrence rates compared with traditional open surgery. In the case of recurrent tumours, a second surgery is considered as the best option if the tumour can be reasonably extirpated. The present study shows a recurrence rate of $2 \%$. The 2 patients had advanced-stage tumours and underwent open surgery. Recurrence was detected in the base of the pterygoid plates and in the infratemporal fossa. Both patients remain free of disease after surgical salvage. Usually JAs recur within 6 to 36 months after primary therapy ${ }^{(2)}$. During 3-year minimum follow-up after treatment, we observed the absence of disease progression in all patients, which confirms the effectiveness of our protocol.

\section{Conclusion}

$\mathrm{JA}$ is a complex disease that may cause important damage in young patients. With improvement of surgical techniques and recent advances in radiologic imaging and embolisation, the management of JAs has changed. Surgery is the main therapeutic option for the treatment of these tumours. We recommend endoscopic resection for early stage tumours (stages I to IIla). Anterior facial translocation through degloving and the subtem- poral-preauricular approach allow performing radical surgery with moderate sequelae in advanced tumours (stages IIIb to IV). Radiosurgery after surgery seems to be a valuable option in the long-term control of JN involving vital structures. These tumours should be treated at centres with expertise in skull base surgery to achieve the best results in terms of complete surgical resection and low morbidity. Our protocol offers long-term cure with acceptable morbidity.

\section{Authorship contribution}

Drs López, Llorente, V. Suárez, Costales and C. Suárez had full access to all the data in the study and take responsibility for the integrity of the data and the accuracy of the data analysis. Study concept and design: López, C. Suárez and Llorente. Acquisition of data: López, Costales and V. Suárez. Analysis and interpretation of data: López and Llorente. Drafting of the manuscript: López and Llorente. Critical revision of the manuscript for important intellectual content: López, Llorente and C. Suárez. Study supervision: Llorente.

\section{Conflict of interest}

None of the authors have financial interests in companies or other entities that have an interest in the information in the contribution.

\section{References}

1. Coutinho-Camilo CM, Brentani MM. Genetic alterations in juvenile nasopharyngea angiofibromas. Head Neck. 2008; 30: 390 400.

2. Renkonen S, Hagström J, Vuola J, et al. The changing surgical management of juvenile nasopharyngeal angiofibroma. Eur Arch Otorhinolaryngol. 2011; 268: 599-607.

3. Midilli R, Karci B, Akyildiz S. Juvenile nasopharyngeal angiofibroma: analysis of 42 cases and important aspects of endoscopic approach. Int J Pediatr Otorhinolaryngol. 2009; 73: 401-408.

4. Fagan JJ, Snyderman CH, Carrau RL, et al, Nasopharyngeal angiofibromas: selecting a surgical approach. Head Neck. 1997; 19: 391-399.

5. Lund VJ, Stammberger $H$, Nicolai $P$, Castelnuovo $\mathrm{P}$ on behalf of the European Rhinologic Society Advisory Board on Endoscopic Techniques in the Management of Nose, Paranasal Sinus and Skull Base Tumors. Rhinol Suppl. 2010: 1: 1-143.

6. Snydermann $\mathrm{CH}$, Pant H, Carrau RL, Gadner P. A new endoscopic staging system for angiofibromas. Arch Otolaryngol Head Neck Surg. 2010; 136: 588-594.

7. Lloyd G, Howard D, Lund VJ, Savy L. Imaging for juvenile angiofibroma. J Laryngol Otol. 2000; 114: 727-730.

8. Li JR, Qian J, Shan XZ, Wang L. Evaluation of the effectiveness of preoperative embolization in surgery for nasopharyngeal angiofi- broma. Eur Arch Otorhinolaryngol. 255: 430-432.

9. Mann WJ, Jecker P, Amedee RG. Juvenile angiofibromas:changing surgical concept over the last 20 years. Laryngoscope. 2004; 114: 291-293.

10. Glad H, Vainer B, Buchwald C, et al. Juvenile nasopharyngeal angiofibromas in Denmark 1981-2003: diagnosis, incidence, and treatment. Acta Otolaryngol. 2007; 127: 292-299.

11. McCombe A, Lund VJ, Howard DJ. Recurrence in juvenile angiofibroma. Rhynology. 1990; 28: 97-102.

12. Mondejar J, Escobar Sánchez C. Complicaciones en la embolización terapéutica del angiofibroma juvenil de rinofaringe. Acta Otorrinolaringol Esp. 1991; 42: 51-53.

13. Scholtz AW, Appenroth E, Kammen-Jolly K, Scholtz LU, Thumfart WF. Juvenile nasopharyngeal angiofibroma: management and therapy. Laryngoscope. 2001; 111: 681-687.

14. Enepekides DJ. Recent advances in the treatment of juvenile angiofibroma. Curr Opin Otolaryngol Head Neck Surg. 2004; 12: 495-496.

15. Janecka IP, Sen CN, Sekhar LN, Arriaga M. Facial translocation: a new approach to the cranial base. Otolaryngol Head Neck Surg. 1990; 103: 413-419.

16. Llorente JL, Nazar G, Cabanillas R, Fernández de León R, Suárez C. Subtemporalpreauricular approach in the management of infratemporal and nasopharyngeal tumours. J Otolaryngol. 2006; 35: 173-179.

17. Hackman $\mathrm{T}$, Snyderman $\mathrm{CH}$, Carrau R, Vescan A, Kassam A. Juvenile nasopharyngeal angiofibroma: The expanded endonasal approach. Am J Rhinol Allergy. 2009; 23: 95-99.

18. Bales C, Kotapka M, Loevner LA, et al. Craniofacial resection of advanced juvenile nasopharyngeal angiofibroma. Arch Otolaryngol Head Neck Surg. 2002; 128: 1071-1078.

19. Lang DA, Neil-Dwyer G, Evans BT, Honeybul S. Craniofacial access in children. Acta Neurochir (Wien). 1998; 140: 33-40.

20. Nicolai P, Berlucchi M, Tomenzoli D. Endoscopic surgery for juvenile angiofibroma: when and how. Laryngoscope. 2003; 113: 775-782.

21. Nicolai $P$, Villaret $A B$, Farina D. Endoscopic surgery for juvenile angiofibroma: a critical review of indications after 46 cases. Am J Rhinol Allergy. 2010; 24: e67-72.

22. Hofmann T, Bernal-Sprekelsen M, Koele W, Reittner $\mathrm{P}$, Klein E, Stammberger $\mathrm{H}$. Endoscopic resection of juvenile angiofibromas long term results. Rhinology. 1995; 43: 282-289.

23. Pryor SG, Moore EJ, Kasperbauer JL. Endoscopic versus traditional approaches for excision of juvenile nasopharyngeal angiofibroma. Laryngoscope. 2005; 115: 1201-1207.

24. Riggs S, Orlandi RR. Juvenile nasopharyngeal angiofibroma recurrence associated 
with exogenous testosterone therapy. Head Neck. 2010; 32: 812-815.

25. Goepfert H, Cangir A, Lee YY. Chemotherapy for aggressive juvenile nasopharyngeal angiofibroma. Arch Otolaryngol. 1985; 111 : 285-289.

26. McAfee WJ, Morris CG, Amdur RJ, et al. Definitive radiotherapy for juvenile nasopharyngeal angiofibroma. Am J Clin Oncol. 2006; 29: 168-170.

27. Mishra SC, Shukla GK, Bhatia N, Pant MC. A rational classification of angiofibromas of the postnasal space. J Laryngol Otol. 1989; 103: 912-916.

28. Reddy KA, Mendenhall WM, AMdur RJ, et al. Long-term results of radiation therapy for juvenile nasopharyngeal angiofibroma. Am J Otolaryngol. 2001; 22: 172-175.
29. Lee JT, Chen P, Safa A, et al. The role of radiation in the treatment of advanced juvenile angiofibroma. Laryngoscope. 2002; 112: 1217-1220

30. Danesi G, Panizza B, Mazzoni A, Calabrese V. Anterior approaches in juvenile nasopharyngeal angiofibromas with intracranial extension. Otolaryngol Head Neck Surg. 2000; 122: 277-283.

31. Mattei TA, Nogueira GF, Ramina R. Juvenile nasopharyngeal angiofibroma with intracranial extension. Otolaryngol Head Neck Surg. 2011 May 13. [Epub ahead of print]

32. Onerci TM, Yucel OT, Ogretmenoglu O. Endoscopic surgery in treatment of juvenile nasopharyngeal angiofibroma. Int J Pediatr Otolaryngol. 2003, 67: 1219-1225.

33. Howard DJ, Lloyd G, Lund V. Recurrence and its avoidance in juvenile angiofibroma. Laryngoscope. 2001; 111: 1509-1511.

Fernando López Álvarez

C/ Marcos Peña Royo, 20 - 4 A

33013 Oviedo - Asturias

Spain

Tel: +985253607

E-mail: flopez_1981@yahoo.es 